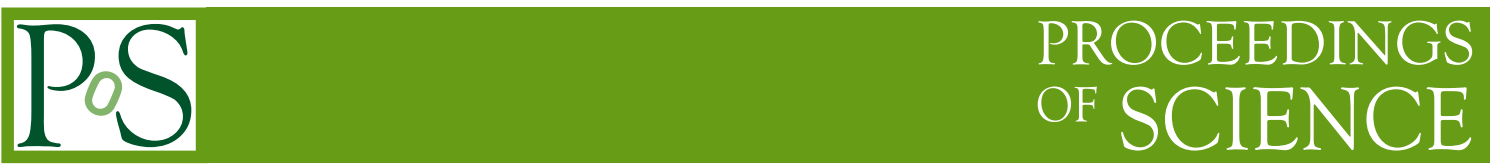

\title{
The KM3NeT neutrino telescopes: status and perspectives
}

\author{
Simone Biagi, on behalf of the KM3NeT collaboration ${ }^{a, *}$ \\ ${ }^{a}$ INFN, Laboratori Nazionali del Sud (LNS), \\ Via S. Sofia 62, Catania, Italy \\ E-mail: biagi@infn.it
}

The KM3NeT international collaboration has started to build two underwater neutrino telescopes, located in two deep sites of the Mediterranean Sea. ARCA (Astroparticle Research with Cosmics in the Abyss) in its final configuration will instrument 1 Gton of seawater, using more than 100,000 PMTs with a 3" diameter. ARCA is optimised to detect cosmic neutrinos within an energy range of $1 \mathrm{TeV}-10 \mathrm{PeV}$; it will provide an excellent view of the Southern Sky, including the Galactic Centre. ORCA (Oscillation Research with Cosmics in the Abyss) will be a smaller detector, with an instrumented volume of a few Mtons. The photosensors are distributed in a more compact lattice for ORCA, in order to reveal atmospheric neutrinos in the $1-100 \mathrm{GeV}$ range.

ORCA is running in a 6-line configuration since more than one year. ARCA has recently completed the installation of new lines, bringing the total number of active lines to 8. The 14 Detection Units of ARCA and ORCA represent the first core towards full construction of KM3NeT, with new deployment campaigns foreseen in the next months and years at the two installation sites.

These proceedings will focus on the status and the long-term perspectives for the completion of the ARCA detector, together with a description of the main technological solutions adopted. The ARCA science program for neutrino astronomy will be presented. Finally, a preliminary analysis of the ARCA 6-line data will be discussed.

\footnotetext{
*** The European Physical Society Conference on High Energy Physics (EPS-HEP2021), ***

*** 26-30 July $2021 * * *$

*** Online conference, jointly organized by Universität Hamburg and the research center DESY ***
}

\footnotetext{
${ }^{*}$ Speaker
} 


\section{Physics goals}

The study of the neutrino has a central role in contemporary physics, since more than 50 years. Neutrino properties (like their masses, the oscillation parameters, the existence of sterile neutrinos, etc...) are not fully determined. New physics will certainly emerge with future discoveries in the neutrino field. In addition to that, neutrino astronomy is nowadays a well defined field in astroparticle physics [1]. In recent years, multimessenger astronomy has started to play an emerging role in cosmological studies. The possibility of joint detections using different mediators, like photons, gravitational waves, high energy cosmic rays, and neutrinos, has already opened new perspectives in cosmic and astronomical studies [2].

In this open field, the KM3NeT collaboration wants to play an important role, currently focusing its activities on the construction of two big underwater neutrino detectors, in the Mediterranean Sea. The ORCA detector (Oscillation Research with Cosmics in the Abyss) will explore the neutrino oscillation parameters, through the detection of atmospheric neutrinos in the energy range $1-100 \mathrm{GeV}$. More details about ORCA are given in [3].

Neutrino astronomy is the main goal of the ARCA telescope (Astroparticle Research with Cosmics in the Abyss), which is designed to reveal cosmic neutrinos in the energy range $1 \mathrm{TeV}-10 \mathrm{PeV}$. The detection and extensive study of astrophysical neutrinos will provide unique information to understand the mechanisms that produce Cosmic Rays in the most energetic phenomena in the Universe. Given their neutral electrical charge, neutrinos point back to their source, allowing to study the astrophysical object using neutrinos as a probe. Finally, the detection of cosmic neutrinos is the "smoking gun" that hadronic processes contribute directly to the Cosmic Ray acceleration.

There are three main channels that neutrino telescopes exploit for event selection. The first is the search for a cosmic diffuse neutrino flux. Neutrino events are collected without any assumption on their directions; an excess of events above the atmospheric neutrino flux is searched for at high energies $(\gtrsim 50 \mathrm{TeV})$ [4]. The second channel is the search for point-like sources in the sky. It is expected to observe a statistical accumulation of events around well defined positions in the skymap [5]. Finally, a multi-messenger approach can be applied to neutrino studies, searching for space-time coincidences with the signals reported by other telescopes that use different probes.

\section{Detector technology}

ARCA is located in the Mediterranean Sea at $3500 \mathrm{~m}$ depth, $80 \mathrm{~km}$ South-East the Sicilian coasts of Portopalo di Capo Passero, Italy. To maximise the detection efficiency, huge volumes of a transparent medium must be used as the active part of the detector. Using an array of photomultiplier tubes (PMTs), it is possible to detect the Cherenkov light induced by secondary charged particles generated by neutrino interactions inside or in the vicinity of the apparatus. The medium used by ARCA is the deep sea water, which has the advantage of very small light scattering, improving the angular resolution of the telescope. The natural optical background is mainly due to the ${ }^{40} \mathrm{~K}$ decay in marine water and to bioluminescence, but considering the different energies that characterise these events with respect to the cosmic neutrinos this background can be easily taken under control. Being located in the Northern Hemisphere, ARCA provides a complementary view to IceCube 


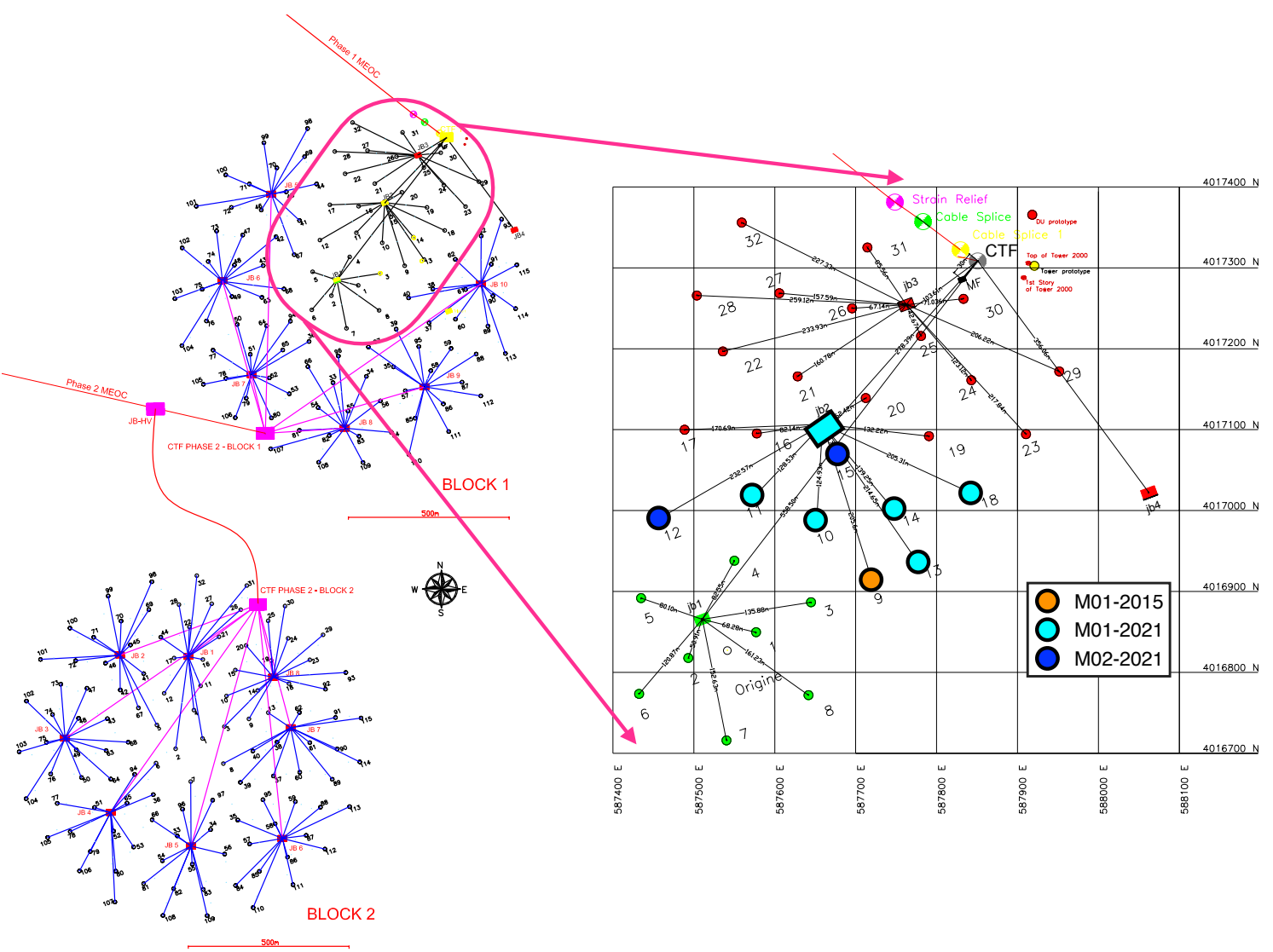

Figure 1: Left: Full KM3NeT-ARCA layout with two building blocks, each of them composed of 115 detection units. Right: The zoom represents the so-called Phase-I of the project, with 31 Detection Units and 1 Calibration Unit. The coloured points stand for the 8 active DUs that are currently taking data in ARCA; each colour represent a different sea campaign.

(located at the South Pole), and has the Milky Way in its field of view, where the presence of the Galactic Centre should guarantee an interesting population of galactic sources.

The complete ARCA layout foresees the construction and installation in the deep waters of two building blocks, that together will instrument an active volume of the order of $1 \mathrm{~km}^{3}$, i.e. 500 Mton of water per block [6]. Each block will be composed of 115 Detection Units (DUs), with an interspacing between DUs of $\sim 90 \mathrm{~m}$. In Fig. 1 Left it is shown the complete ARCA footprint. Two Main Electro-Optical Cables (MEOC) $100 \mathrm{~km}$-long are already installed onsite and provide connectivity to an onshore laboratory, property of INFN (Istituto Nazionale di Fisica Nucleare, Italy). Offshore, the two MEOCs will be connected to a network of junction boxes and inter-link cables to distribute power to the DUs and allow high-rate data transfer via optical fibres.

The Detection Unit is the basic element of the ARCA telescope. It is a floating vertical structure, anchored on the sea bottom and kept in position by buoys on top, see Fig. 2 Right. In the ARCA setup, the DU is $700 \mathrm{~m}$ heigh, and allocates 18 Digital Optical Modules (DOMs), with an inter-DOM spacing of $36 \mathrm{~m}$. Each DOM is composed of a 17" glass sphere that hosts 31 PMTs of 3", as well as the power and control electronics, see Fig. 2 Left. The DOM design allows for digital 

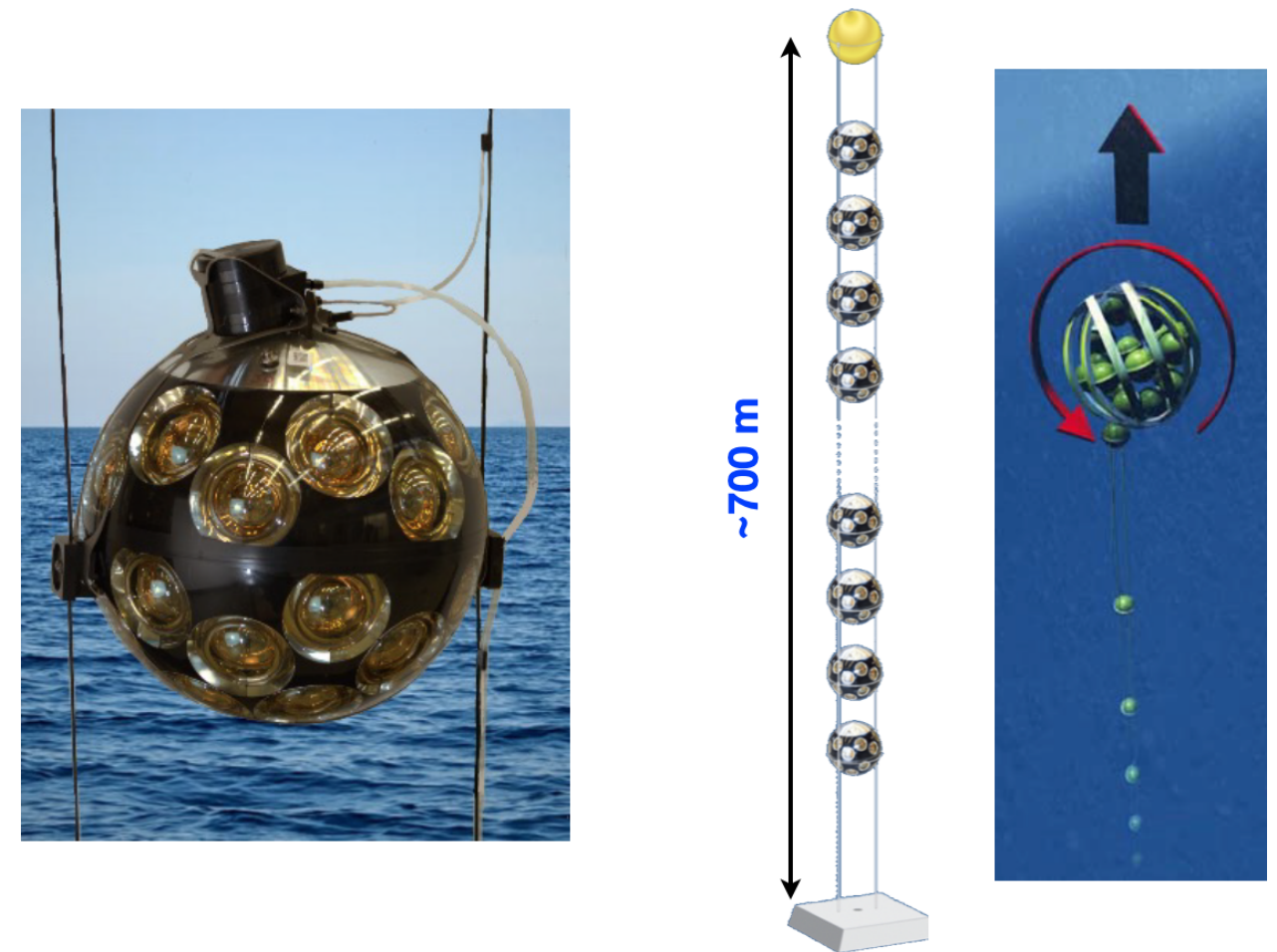

Figure 2: Left: A picture of the Digital Optical Module mounted on a Detection Unit. Right: A sketch of a Detection Unit and a drawing that shows the self-unrolling installation underwater.

photon counting, useful for energy determination, and naturally provides directional information that can improve the detector angular resolution. The data taking strategy is based on the "all data to shore" approach, that takes advantage of the large bandwidth ( $\sim \mathrm{Gbit} / \mathrm{s})$ provided by optical fibres. Data are packed using the WhiteRabbit protocol, then transferred onshore where they are triggered, time-calibrated, reconstructed, filtered, and written on disk [7].

\section{ARCA6 and ARCA8 data}

Even if still under construction, ARCA is conceived to be operated since the first DU is deployed and connected to the submarine network. In fact, besides the growth of the underwater detector, also the onshore infrastructure is being updated following the evolution of the detector. With a live time that is $>99 \%$, data are continuously retrieved onshore and triggered according to the detector configuration.

The first DU was deployed in December 2015, with a further two DUs in May 2016; these two DUs were recovered later as they ceased to work. After an interruption in the construction due to problems with the submarine network, other five DUs were installed in April 2021, and the last two DUs were deployed in September 2021. Using data taken in 2016 - 2017 with two ARCA DUs and one ORCA DU, it was possible to measure the atmospheric muon flux in a wide range between $2230-3390 \mathrm{~m}$ seawater depth [8]. This first result demonstrated the goodness of the data analysis procedures, including calibrations, and showed the potential of combining data from the two detectors. 

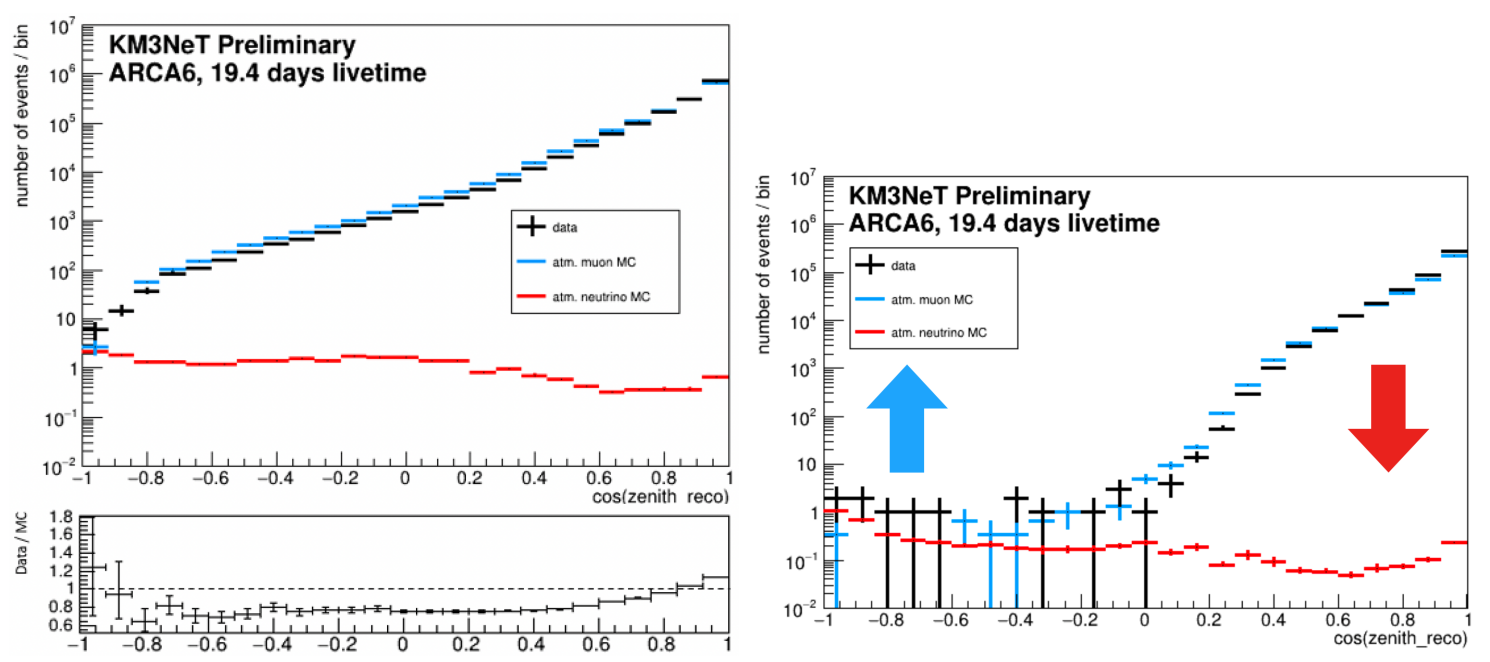

Figure 3: Number of selected events for 19.4 days of ARCA6 data as a function of the reconstructed zenith angle [10]. Data are compared with Monte Carlo simulations. Left: Loose quality cuts applied; atmospheric muons dominate the sample of atmospheric neutrinos. Right: Quality cuts applied to the reconstruction parameters in order to select atmospheric neutrino candidates. Only genuine neutrino tracks can have a zenith angle $>90 \mathrm{deg}$.

Some preliminary results were produced analysing ARCA6 data, and are summarised in Fig. 3. Muon track events are triggered looking at local coincidences between PMTs in a DOM, in a time window typically $=20 \mathrm{~ns}$. Then, trigger algorithms search for space-time correlations between DOMs, considering two main event topologies: track-like and shower-like events. The former is dominated by charged current interactions of muon neutrinos, that generate a secondary muon that can travel for $\mathrm{km}$ in the water, producing the typical Cherenkov light cone. The latter is produced with neutral current interactions of neutrinos of any flavour, together with charge current interactions of electron and tau neutrinos. The golden channel for neutrino astronomy is track-like, as the angular resolution in this case is estimated to be better than $0.2 \mathrm{deg}$ at energies above $10 \mathrm{TeV}$, with the complete ARCA detector [6]. A sample of 19.4 days of data is used in this preliminary analysis, together with Monte Carlo (MC) samples of atmospheric muons and neutrinos. The Monte Carlo simulation chain includes: event generation (neutrinos and amospheric muons), particle propagation, Cherenkov light production and propagation in water, and data acquisition simulation [9]. At the final step, both data and MC events are reconstructed using the same algorithms, and then compared.

The reconstructed data sample with loose quality cuts is plotted as a function of the reconstructed zenith angle in Fig. 3 Left. Monte Carlo atmospheric muons and neutrinos are reported for comparison. Only neutrinos can produce genuine up-going events (i.e. zenith $>90 \mathrm{deg}$ ). As the event rate of muons is several orders of magnitude higher than the rate from neutrinos, even a small fraction of mis-reconstructed muons overcome completely the neutrino events. This plot shows a data-MC agreement at the level of $20 \%$, which demonstrates that the main systematics are under control and that the space-time calibration is properly applied.

With some additional requirements on the quality parameters of the reconstruction, it is possible to reduce the data and MC samples with the purpose of getting rid of atmospheric muons and select 


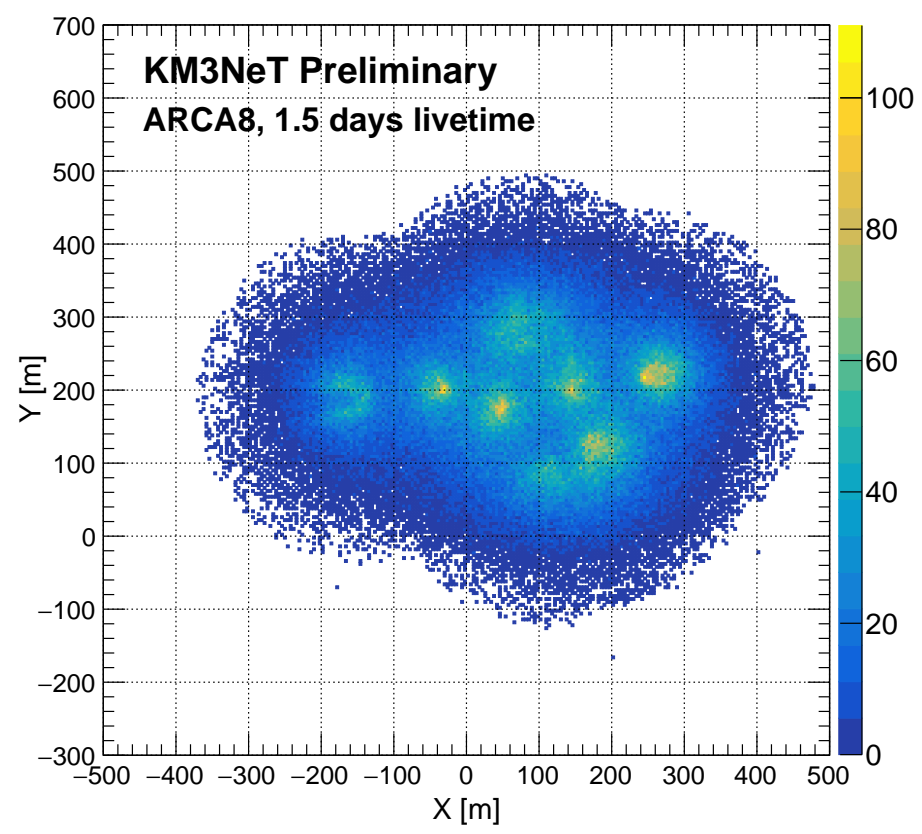

Figure 4: $\mathrm{X}$ and $\mathrm{Y}$ coordinates of the estimated starting point of light emission for reconstructed tracks of atmospheric muons in ARCA8. Tracks with more than 10 hits are selected. The footprint of the 8 DUs can be qualitatively derived.

a "pure" neutrino sample in the up-going sector. This is shown in Fig. 3 Right, where the 15 events are observed as up-going $\left(\cos \theta_{\text {reco }}<0\right)$, where MC has 4 events from atmospheric $v$ and 7 events form atmospheric $\mu$. Selected data are statistically compatible with MC expectations. More details on the neutrino selection analysis can be found in [10].

Adding the recently deployed two DUs to the sample, some days of data were collected in the ARCA8 configuration. To check if positioning and timing is properly working, a simple plot can be produced, see Fig. 4. In this, reconstructed track-like events are selected with the minimal request of having at least ten hits. The algorithm that reconstructs track-like events provides the estimated starting point of each track in the fiducial volume of the detector. Fig. 4 is a 2-D histogram filled with the $\mathrm{X}$ and $\mathrm{Y}$ coordinates of the estimated starting points of light emission, where points accumulate around the nominal positions of the eight working Detection Units. A nice footprint of ARCA8 appears. In fact, muons passing through or very close to a DOM will be reconstructed with higher efficiency, thus their starting point of the track will be reconstructed close to the DUs.

\section{Next steps}

The KM3NeT collaboration has started the mass production of the ARCA and ORCA underwater detectors. The collaboration aims at the completion of ARCA Phase-I before the end of 2022, which will comprise 31 Detection Units plus a Calibration Unit. The long-term plan currently in place foresees to complete the two building blocks before the end of 2030, but an intense management activity is ongoing in order to accelerate this process and reduce the building phase. 


\section{References}

[1] M.G. Aartsen et al. (IceCube Collaboration), Evidence for high-energy extraterrestrial neutrinos at the IceCube detector, Science 342 (2013) 947.

[2] A. Albert et al., Search for multimessenger sources of gravitational waves and high-energy neutrinos with Advanced LIGO during its first observing run, ANTARES, and IceCube, ApJ 870 (2019) 134.

[3] R. Shanidze, KM3NeT/ORCA overview, these proceedings.

[4] A. Albert et al. (ANTARES Collaboration), All-flavor search for a diffuse flux of cosmic neutrinos with 9 years of ANTARES data, ApJL 853 (2018) L7.

[5] S. Aiello et al. (KM3NeT Collaboration), Sensitivity of the KM3NeT/ARCA neutrino telescope to point-like neutrino sources, Astropart. Phys. 111 (2019) 100.

[6] S. Adrián-Martínez et al. (KM3NeT Collaboration), Letter of intent for KM3NeT 2.0, J. Phys. G: Nucl. Part. Phys. 43 (2016) 084001.

[7] S. Biagi et al. (for the KM3NeT Collaboration), The data acquisition system of the KM3NeT detector, PoS(ICRC2015)1172 (2015).

[8] M. Ageron et al. (KM3NeT Collaboration), Dependence of atmospheric muon flux on seawater depth measured with the first KM3NeT detection units, Eur. Phys. J. C 80 (2020) 99.

[9] A. Albert et al. (ANTARES Collaboration), Monte Carlo simulations for the ANTARES underwater neutrino telescope, JCAP 01 (2021) 064.

[10] A. Sinopoulou et al. (for the KM3NeT Collaboration), Atmospheric neutrinos with the first detection units of KM3NeT/ARCA, PoS(ICRC2021)1134 (2021). 